\title{
Effect of nucleopolyhedrovirus infection on antibacterial activity and antibacterial peptide production in larvae of the silkworm, Bombyx mori (Lepidoptera: Bombycidae)
}

\author{
Seiichi Furukawa, ${ }^{1}$ Aki Sagisaka, ${ }^{1}$ Hiromitsu Tanaka ${ }^{1}$ and Minoru Yamakawa ${ }^{1,2, *}$ \\ ${ }^{1}$ Innate Immunity Research Unit, National Institute of Agrobiological Sciences; Tsukuba 305-8634, Japan \\ ${ }^{2}$ Graduate School of Life and Environmental Sciences, University of Tsukuba; Tsukuba 305-8572, Japan \\ (Received 25 May 2006; Accepted 27 September 2006)
}

\begin{abstract}
Effects of nucleopolyhedrovirus (NPV) on antibacterial peptide gene expression and peptide production in Bombyx mori larvae remain unclear. Antibacterial activity was first examined with the hemolymph from $B$. mori larvae infected with B. mori NPV (BmNPV) and injected with bacterial lipopolysaccharide (LPS) $72 \mathrm{~h}$ postinfection (p.i.). No antibacterial activity was detected. Expression of four antibacterial peptide genes were next analyzed in $B$. mori larvae infected with BmNPV. Although antibacterial peptide genes were activated upon injection of LPS in the larvae infected with BmNPV, none were triggered by BmNPV alone. Production of the antibacterial peptide cecropin B was next examined by Western blotting. Cecropin B was not detected from 72 to $96 \mathrm{~h}$ p.i. The absence of cecropin B was coincident with an increase in BmNPV cysteine proteinase (CP) activity. Cecropin B production was confirmed at $96 \mathrm{~h}$ p.i. in the larvae infected with CP-deficient BmNPV, indicating that the antibacterial peptides are degraded by BmNPV-CP at a very late stage of viral infection. These results suggest that BmNPV neither activates nor suppresses gene expression of antibacterial peptides and that absence of antibacterial activity is due to BmNPV-CP in $B$. mori larvae.
\end{abstract}

Key words: Bombyx mori; NPV; gene expression; peptide production; antibacterial peptides

\section{INTRODUCTION}

Antibacterial responses in the insect immune system have been the most extensively studied (Hoffmann et al., 1999; Stöven et al., 2000; De Gregorio et al., 2002). On the other hand, insect immunity against viral pathogens still remains obscure (Washburn et al., 1996). Of many viruses, Bombyx mori nucleopolyhedrovirus (BmNPV) is the most important virus in the sericultural industry, because farmers often suffer severe economic losses caused by this virus. Nonetheless, studies on immune responses of the silkworm against BmNPV are still in their infancy.

The immune reactions against NPV can be divided into two phases depending on the infection cycle: primary defense against occlusion-derived virus (ODV) in the alimentary canal and secondary defense against the budded virus (BV) in other tis- sues such as the fat body, trachea and hemocytes. Recently, a lipase and a serine protease from the digestive juice of $B$. mori larvae have been demonstrated to show antiviral activity against BmNPV (Ponnuvel et al., 2003; Nakazawa et al., 2004). These two digestive enzymes are speculated to play an important role in the primary defense. Concerning the secondary defense stage, it has been shown that apoptosis is initiated during NPV replication in insect cells and specific viral gene products, P35 and $\mathrm{P} 49$, are responsible for blocking the apoptotic response (Clem et al., 1991; Zoog et al., 2002). Moreover, it was previously demonstrated in the giant silk moth Hyalophora cecropia that an Autographa californica baculovirus (AcNPV) neither activates NF- $\kappa \mathrm{B}$ nor induces Cecropin, Attacin or Lysozyme gene expression (Sun and Faye, 1992). In addition, it has been reported that gene expression of hemolin, one hemolymph protein, is induced by

* To whom correspondence should be addressed at: E-mail: yamakawa@nias.affrc.go.jp DOI: 10.1303/aez.2007.55 
NPV infection in the Chinese oak silk moth $A n$ theraea pernyi and that no Attacin gene expression or antibacterial activity is observed (Hirai et al., 2004).

We have concentrated our studies on antibacterial peptides from B. mori (Yamakawa and Tanaka, 1999). Some antibacterial peptides are known to not only have antibacterial properties but also antifungal and antiparasitical properties (Kimbrell, 1991). However, whether antibacterial peptides are involved in defense reactions against viral infection has not been extensively analyzed. In this study, we focused on the effects of BmNPV on gene expression of $B$. mori antibacterial peptides to determine if BmNPV infection causes up or down regulation of these peptides genes. We also investigated whether BmNPV induces or shuts down the production of cecropin B by using antibodies against the peptide.

\section{MATERIALS AND METHODS}

Insects. $B$. mori larvae (Tokai $\times$ Asahi strain) were reared on artificial diet (Nihonnosanko) at $25^{\circ} \mathrm{C}$ under a controlled environment $(11 \mathrm{~h}$ light and $13 \mathrm{~h}$ dark). Fifth instar larvae (1 d) were used in the experiments.

Infection of $B$. mori larvae with BmNPV-BV, injection of lipopolysaccharide (LPS) and collection of hemolymph. Larvae were first infected with BmNPV-BV $\left(8 \times 10^{5} \mathrm{TCID}_{50} /\right.$ larva $)$. Naive larvae were also used as controls. Lipopolysaccharide (Sigma, $20 \mu \mathrm{g} /$ larva) was injected into the larvae $72 \mathrm{~h}$ postinfection (p.i.). As a control saline instead of LPS was injected. Larvae were kept at $25^{\circ} \mathrm{C}$ for $24 \mathrm{~h}$. Hemolymph was collected by cutting off a leg into ice-cooled tubes containing phenylthiourea (Sigma, $50 \mu \mathrm{g} / \mathrm{ml}$ ). For kinetic experiments, hemolymph was collected $0,4,8,12$ and $24 \mathrm{~h}$ after LPS injection from larvae infected with BmNPV ( $72 \mathrm{~h}$ p.i.). Hemolymph was heated at $95^{\circ} \mathrm{C}$ for $15 \mathrm{~min}$ and centrifuged at $10,000 \times g$ for $5 \mathrm{~min}$

Antibacterial activity assay. Plate-growth-inhibition assay was according to reported methods (Hultmark et al., 1980). Escherichia coli XL1-Blue $\mathrm{MRF}^{\prime}$ was used as the test bacteria for antibacterial activity assay. Bacteria were cultured at $37^{\circ} \mathrm{C}$ in Luria-Bertani (LB) broth (Sambrook and Russel, 2001). Mid-exponential-phase cultured cells were centrifuged, washed once in $10 \mathrm{~mm}$ sodium phosphate buffer ( $\mathrm{pH}$ 7.4) and suspended in the same buffer. Melted LB broth $(10 \mathrm{ml})$ containing agar and $2-6 \times 10^{5}$ mid-exponential-phase cultured bacteria was pored into sterile Petri dishes $(9 \mathrm{~cm}$ diameter). Samples ( $3 \mu \mathrm{l}$ each) were applied to wells ( $2 \mathrm{~mm}$ diameter) in the LB agar plates. The plates were incubated at $37^{\circ} \mathrm{C}$ overnight. Antibacterial activity was detected as clear inhibition zones after incubation.

Northern blot analysis. Total RNA was extracted from the fat body of larvae at different time intervals $(0,4,8,12$ and $24 \mathrm{~h})$ after LPS injection. Northern blot analysis (Sambrook and Russel, 2001) was conducted with $3.5 \mu \mathrm{g}$ of the total RNA and specific probes labeled with DIG-conjugated dUTP (Roche Diagnostics) using cDNAs for cecropin B (Kato et al., 1993), attacin (Sugiyama et al., 1995), lebocin (Chowdhury et al., 1995) or moricin (Furukawa et al., 1997). Polyhedrin gene expression of BmNPV was also analyzed with a specific probe prepared by PCR using BmNPV genomic DNA as a template and forward and reverse primers (5'-AAGCGCAAGAAGCACCTAGT-3' for forward primer and 5'-TGTACTCGCTGTGGATGTTC-3' for reverse primer taken from the genomic sequence data base, GenBank accession no. L33180).

Western blot anaysis. The larvae were first infected with BmNPV-BV or cysteine protease (CP)deficient BmNPV-BV $72 \mathrm{~h}$ before LPS injection. As controls, naive larvae were injected with LPS. Hemolymph was collected at the indicated time intervals $(0,4,8$ and $24 \mathrm{~h})$ after LPS injection. Conditions for BmNPV-BV infection and LPS injection were the same as described above. The hemolymph was heat-treated at $95^{\circ} \mathrm{C}$ for $15 \mathrm{~min}$ and centrifuged at $10,000 \times g$ for $5 \mathrm{~min}$. One microliter of the supernatant was loaded onto the gel with size markers. As a positive control, $1 \mu \mathrm{g}$ of chemically synthesized cecropin B (Kadono-Okuda et al., 1995) was loaded onto the gel. Preparation of polyclonal antibodies against cecropin B from $B$. mori was as reported previously (Nakazawa et al., 2003). Western blotting was conducted as described by Sambrook and Russel (2001).

Measurement of CP activity. $B$. mori larvae were infected with BmNPV-BV and hemolymph collected at different time intervals $(0,24,48,72$ and $96 \mathrm{~h}$ p.i.). Infection conditions were the same 
as described above. Hemolymph was collected into a tube with phenylthiourea to prevent melanization. Activity of CP was measured with $10 \mu \mathrm{l}$ of the hemolymph using Azocoll (Sigma) as a substrate according to the methods of Kobayashi et al. (1985).

Degradation of cecropin $B$ in the hemolymph from larvae infected with BmNPV-BV. B. mori larvae were infected with BmNPV-BV or CP-deficient BmNPV-BV and hemolymph was collected at different time intervals $(24,48,72$ and $96 \mathrm{~h})$. The hemolymph samples $(6 \mu \mathrm{l}$ each) were mixed with hemolymph $(1 \mu \mathrm{l})$ containing endogenous cecropin $\mathrm{B}$ from larvae $12 \mathrm{~h}$ after injection with LPS (20 $\mu \mathrm{g} /$ larva). The mixtures were incubated at room temperature for 1,4 and $16 \mathrm{~h}$, then electrophoresed and Western blotting conducted.

\section{RESULTS}

\section{Effect of BmNPV infection on the induction of antibacterial activity}

Antibacterial activity against $E$. coli of the hemolymph from $B$. mori larvae infected with BmNPV-BV and then injected with saline instead of LPS was analyzed first by the inhibition zone assay. Antibacterial activity was not detected in the larvae infected with BmNPV-BV (Fig. 1A), showing BmNPV does not induce activity. Antibacterial activity was next examined using hemolymph from larvae infected with BmNPV-BV and then injected $72 \mathrm{~h}$ p.i. with LPS, which may be contaminated with peptidoglycan. The hemolymph showed strong antibacterial activity at 12 and $24 \mathrm{~h}$ (84 and $96 \mathrm{~h}$ p.i., respectively) after LPS injection (Fig. 1A), indicating normal induction of antibacterial activity by LPS in the uninfected larvae but not in the BV-infected larvae. A time course for the induction of antibacterial activity was next investigated in the larvae infected with BmNPV. The results indicated that the hemolymph from larvae infected with BmNPV showed no antibacterial activity, although uninfected control samples showed a typical pattern of antibacterial activity with time after LPS injection (Fig. 1B). These results suggest two possibilities. 1) Gene expression and synthesis of antibacterial peptides by LPS were inhibited in the larvae 76-96h p.i. or 2) antibacterial peptides induced by LPS were degraded in the larvae 7696 h p.i.

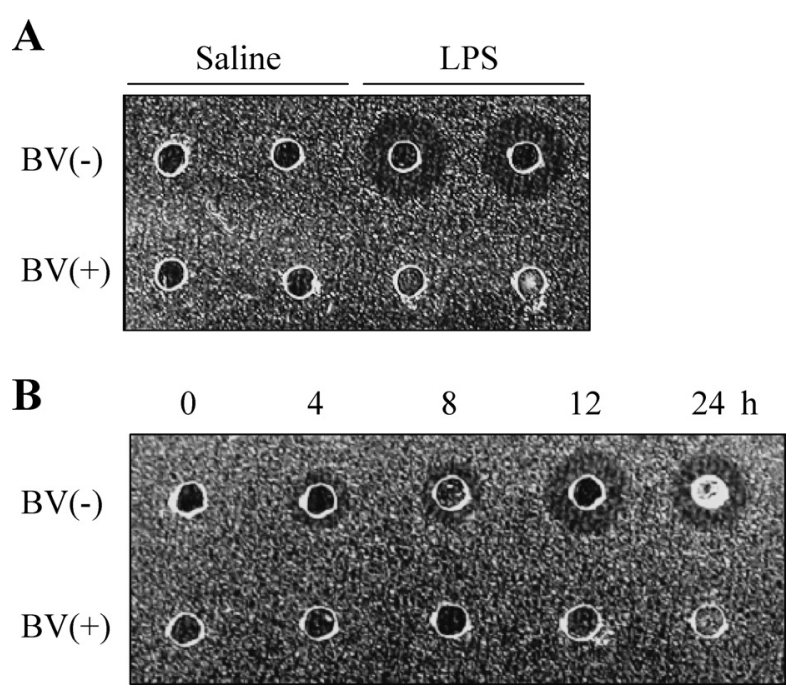

Fig. 1. Effect of BmNPV infection on the induction of antibacterial activity in larvae. Panel A: Antibacterial activity in larvae infected with BmNPV. B. mori larvae were infected with BmNPV and then LPS or saline was injected into the larvae $72 \mathrm{~h}$ p.i. As a control, larvae were injected with saline instead of BmNPV. BV $(+)$ and $\mathrm{BV}(-)$ denote larvae infected with BmNPV or injected with saline, respectively. Panel B: Time course of induction of antibacterial activity in larvae infected with BmNPV $(\mathrm{BV}(+))$. Naive larvae $(\mathrm{BV}(-))$ were used as controls. Hemolymph was collected 0, 4, 8, 12 and $24 \mathrm{~h}(72,76,80,84$ and 96 h p.i., respectively) after LPS injection.

\section{Effect of BmNPV infection on expression of an- tibacterial peptide genes}

Whether gene expression and production of antibacterial peptides were inhibited or these peptides were degraded were next examined. Gene expression of four B. mori antibacterial peptides that show antibacterial activity against $E$. coli was first analyzed by Northern blotting in larvae infected with BmNPV. Total RNA was extracted from the fat body, which is the main tissue for expression of antibacterial peptide genes (Kato et al., 1993; Chowdhury et al., 1995; Sugiyama et al., 1995; Taniai et al., 1996; Furukawa et al., 1997, 1999). Although no activation of antibacterial peptide genes encoding cecropin B (Kato et al., 1993; Taniai et al., 1995), attacin (Sugiyama et al., 1995), lebocin (Chowdhury et al., 1995; Furukawa et al., 1997) and moricin (Furukawa et al., 1999) by BmNPV was observed, these peptide genes were induced normally with time $(4,8,12$ and $24 \mathrm{~h})$ by LPS in the larvae that were infected with BmNPV $72 \mathrm{~h}$ before LPS injection (Fig. 2). Under these conditions polyhedrin gene was expressed nor- 


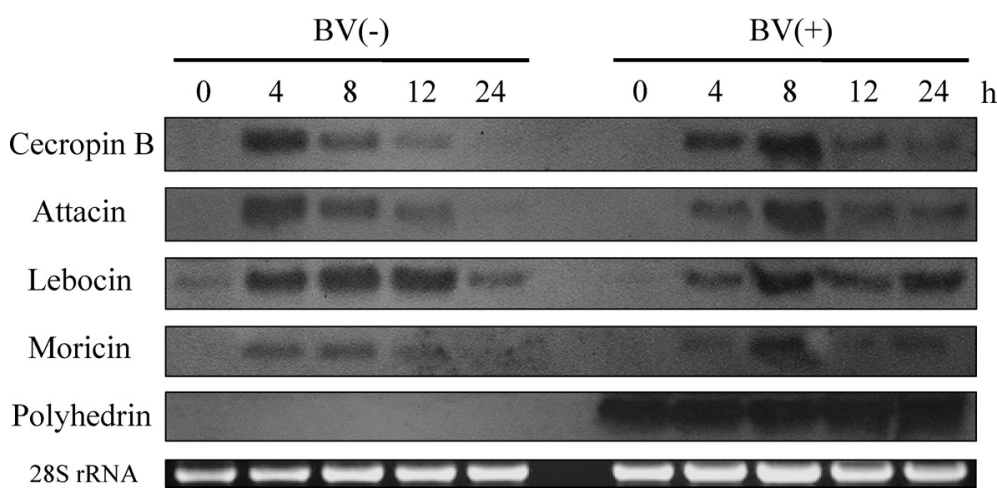

Fig. 2. Expression of $B$. mori antibacterial peptide genes in larvae infected with BmNPV. B. mori larvae were first infected with BmNPV and then LPS was injected into the larvae $72 \mathrm{~h}$ p.i. Total RNA was extracted from the fat body of the larvae 0, 4, 8, 12 and $24 \mathrm{~h}(72,76,80,84$ and $96 \mathrm{~h}$ p.i., respectively) after LPS injection. Northern blot analysis was conducted with the total RNA and specific probes. Polyhedrin gene expression of BmNPV was also analyzed. As an internal control, 28S rRNA was visualized with ethidium bromide. BV(+): Larvae infected with BmNPV-BV. BV(-): Larvae injected with saline.

mally and BmNPV itself showed no ability to activate antibacterial peptide genes. The induction of these four antibacterial peptide genes was also observed in the uninfected control larvae (Fig. 2). These results indicated that expression of the four antibacterial peptide genes are not affected by BmNPV in the larvae 76-96h p.i., suggesting that down-regulation of antibacterial peptide genes by BmNPV infection does not occur in the larvae at the late stage of infection.

\section{Effect of BmNPV infection on the production of cecropin B}

Of four types of antibacterial peptides from $B$. mori, cecropin B is the only peptide for which an antibody is available (Nakazawa et al., 2003). Therefore, we focused on cecropin B to clarify the effects of BmNPV on the production of antibacterial peptides. Western blot analysis was conducted using polyclonal antibodies against cecropin B and hemolymph protein samples from larvae infected with BV. The experimental conditions were the same as the Northern blot analysis. No cecropin B was detected at $0,4,8,12$ and $24 \mathrm{~h}$ post LPS-injection in the larvae infected with BV, whereas hemolymph samples from saline-injected control larvae showed clear signals for cecropin B showing a typical induction pattern of the peptide synthesis, namely a gradual increase up to $12 \mathrm{~h}$ post LPS-injection and a decrease thereafter (Fig. 3). These results suggest either that the protein synthesis shutdown occurs in the larvae $76-96 \mathrm{~h}$ p.i. or that synthesized cecropin B is completely degraded in lar-

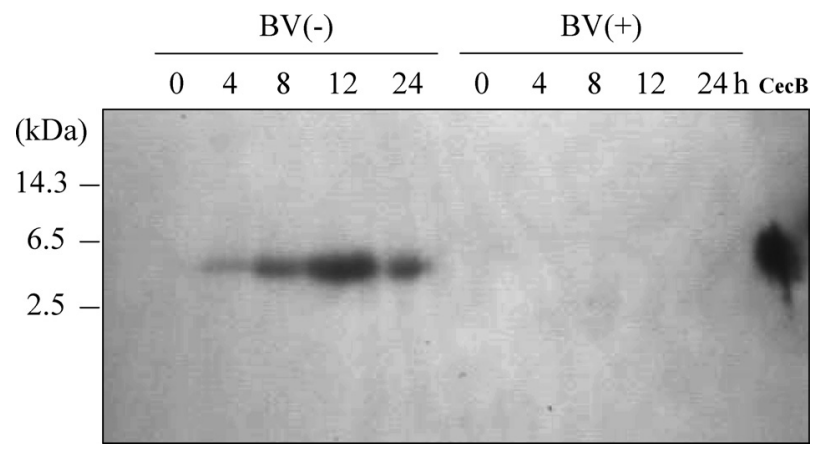

Fig. 3. Western blot analysis of cecropin B in the hemolymph of LPS-injected larvae, which were beforehand infected with BmNPV-BV and LPS was injected $72 \mathrm{~h}$ p.i. Hemolymph was collected $0,4,8,12$ and $24 \mathrm{~h}(72,76,80,84$ and $96 \mathrm{~h}$ p.i., respectively) after LPS injection. Conditions for BmNPV-BV infection and LPS injection were the same as in Fig. 2. Western blotting was conducted with the hemolymph collected from larvae infected with BmNPV and then injected with LPS as described in Fig. 2. Naive larvae were used as controls. BV $(+)$ and $\mathrm{BV}(-)$ are the same as in Fig. 2. CecB: Synthetic $B$. mori cecropin B as a size marker.

vae at this infection stage.

\section{Degradation of cecropin B by CP of BmNPV}

Cysteine proteinases of BmNPV and AcNPV are hypothesized from deletion experiments of the viral $\mathrm{CP}$ gene to be involved in the destruction of insect tissues during the later stages of pathogenesis (Ohkawa et al., 1994; Slack et al., 1995). This led us to examine the effect of CP-deficient BmNPV on the synthesis of cecropin B. Cysteine proteinase activity in the hemolymph of larvae infected with BmNPV-BV was first measured to 
clarify the relationship between the increment of $\mathrm{CP}$ activity and the degradation of antibacterial peptides. Hemolymph was collected from larvae 0 , 24, 48, 72 and $96 \mathrm{~h}$ p.i. CP activity sharply increased during 72 to $96 \mathrm{~h}$ p.i. (Fig. 4). On the contrary, hemolymph from larvae injected with saline or infected with CP-deficient BmNPV (Ohkawa et al., 1994) did not show CP activity (Fig. 4), which is consistent with previously reported results (Suzuki et al., 1997). Hemolymph containing endogenous cecropin B was then incubated for 1, 4 or $16 \mathrm{~h}$ in vitro with hemolymph collected from larvae infected with BmNPV or CP-deficient BmNPV at different time intervals (24, 48, 72 and $96 \mathrm{~h}$ p.i.). Degradation was confirmed in the hemolymph collected from larvae $96 \mathrm{~h}$ p.i. with wild type BmNPV but not with CP-deficient BmNPV (Fig. 5), indicating coincidence of the timing of cecropin $\mathrm{B}$ degra-

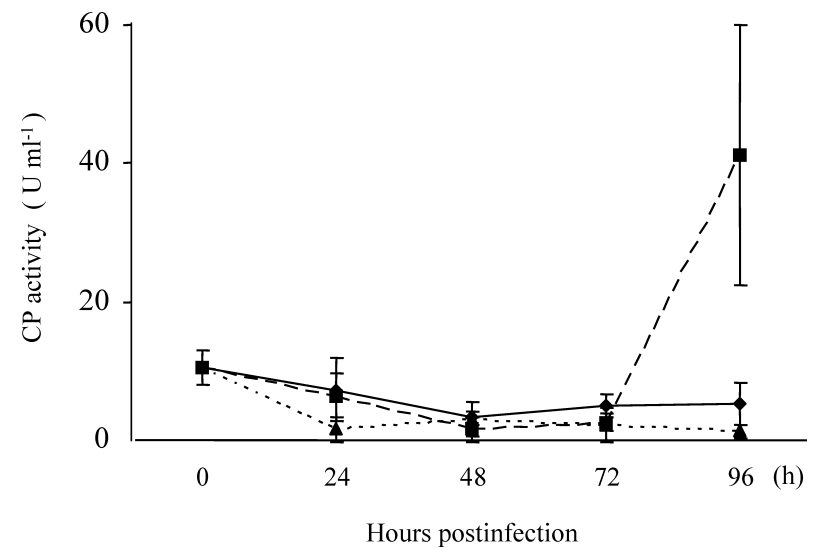

Fig. 4. Kinetics of CP activity in the hemolymph of larvae with BmNPV-BV. The data shown are means \pm SEM from three experiments. B. mori larvae were infected with BmNPV$\mathrm{BV}$ (square) and hemolymph was collected at the indicated time intervals p.i. As controls, larvae were injected with saline (triangle) or infected with CP-deficient BmNPV-BV (diamond). Conditions of infection were the same as in Fig. 2. For other experimental conditions see the Materials and Methods. dation with increased CP activity (Fig. 4). These results suggest that BmNPV-CP possesses the ability to degrade secreted cecropin B in B. mori larvae.

\section{Production of cecropin $B$ at the late stage of in- fection with CP-deficient BmNPV}

Western blot analysis was conducted to examine the production of cecropin $\mathrm{B}$ in $B$. mori larvae at the late stages of the infection (96h p.i.) with CPdeficient BmNPV-BV. The result showed clear signals, although control hemolymph samples infected with wild type BmNPV-BV did not give the signal (Fig. 6). The results suggest that BmNPV$\mathrm{CP}$ degrades the antibacterial peptides directly in the late stages of infection.

\section{DISCUSSION}

Invertebrate immune peptides having antibacterial activity are often reported to have antiviral activity. Tachyplesin I from the horseshoe crab was shown to have antiviral activity against vesicular stomatitis virus (Murakami et al., 1991) and human immunodeficiency virus (HIV) (Morimoto et al., 1991). Melittin and cecropin from insects have been shown to suppress replication of HIV by inhibiting viral gene expression (Wachinger et al., 1998) and alloferon from the blow fly Calliphora vicinia showed antiviral activity against influenza viruses A and B (Chernysh et al., 2002). Although the horseshoe crab and insects are not hosts for these viruses, these results suggest that some invertebrate antibacterial peptides have antiviral activity. However, it remains unclear whether these viruses can induce antibacterial peptide gene expression and peptide production in the horseshoe crab and insects.

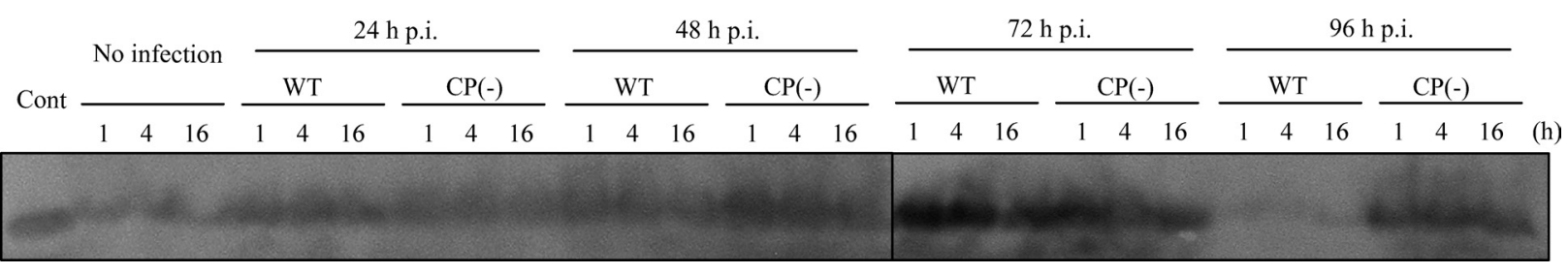

Fig. 5. Effect of the hemolymph from larvae infected with BmNPV-BV on the degradation of cecropin B. B. mori larvae were infected with BmNPV-BV (WT) or CP-deficient BmNPV-BV $(\mathrm{CP}(-))$ and hemolymph was collected at the different time intervals indicated on the top of the panel. The hemolymph samples were mixed with hemolymph containing endogenous cecropin $\mathrm{B}$ and incubated at room temperature for 1, 4 and $16 \mathrm{~h}$. Western blotting was conducted as in Fig. 3. No infection: Hemolymph from uninfected larvae. Cont: Hemolymph sample containing endogenous cecropin B that was not mixed with the BmNPV-BV-infected hemolymph. 


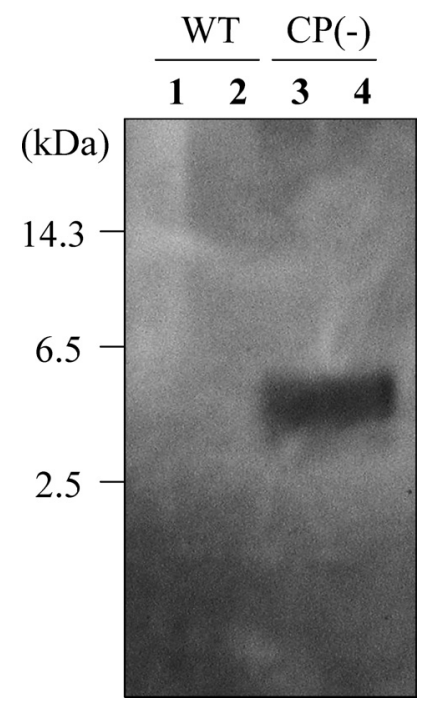

Fig. 6. Production of cecropin B at the late stages of infection with CP-deficient BmNPV. B. mori larvae were infected with CP-deficient BmNPV-BV and then LPS was injected $72 \mathrm{~h}$ p.i. (lanes 3 and 4). Hemolymph was collected from larvae $24 \mathrm{~h}$ after LPS injection. As a control, larvae were infected with wild type BmNPV-BV (lanes 1 and 2). The production of cecropin B was analyzed by Western blotting. For other conditions see the Materials and Methods. WT and $\mathrm{CP}(-)$ are the same as Fig. 5.

Our results indicate that BmNPV neither induces antibacterial activity nor activates antibacterial peptide genes in $B$. mori larvae and that activation of antibacterial peptide genes by LPS in BmNPVinfected larvae are normal. However, degradation of the antibacterial peptide by BmNPV-CP occurs at the very late stage of infection (72-96h p.i.) in B. mori larvae. To our knowledge, this is the first report of the effects of NPV infection on gene expression and production of antibacterial peptides in B. mori larvae. These results are consistent with data that NPV does not activate Attacin genes, nor induce antibacterial activity in $A$. pernyi (Hirai et al., 2004). Taken together with the results on $H$. cecropia (Sun and Faye, 1992) and A. pernyi (Hirai et al., 2004), our results suggest that baculoviruses do not provoke the NF- $\kappa \mathrm{B}$ pathway which plays an important role in antibacterial peptide gene expression in lepidopteran insects. On the other hand, Hirai et al. (2004) speculated that Hemolin gene expression is induced upon virus infection by a pathway that does not involve NF- $\kappa \mathrm{B}$. It is important to examine antiviral activity of $B$. mori antibacterial peptides against BmNPV to determine whether antibacterial peptides are involved in im- mune reactions against BmNPV infection.

In B. mori, comparative expressed-sequence-tag (EST) analysis of differential gene expression profiles was conducted in BmNPV-infected $\mathrm{BmN}$ cells (Okano et al., 2001). The detailed analysis of host derived ESTs showed that expression of most of the major host genes is reduced with BmNPV infection (Okano et al., 2001). However, the expression of the mitochondria-encoded genes, cytochrome b oxidase, cytochrome c oxidase 1 and 3 , is not affected by viral infection (Okano et al., 2001). Consistent with this, a previous report showed that expression of the Spodoptera frugiperda cytochrome oxidase 3 gene was stable until at least $24 \mathrm{~h}$ p.i. during infection with AcNPV (Ooi and Miller, 1988). On the other hand, the ATP/ADP translocase and mitochondrial phosphate carrier protein genes of $B$. mori, that are encoded by the host nuclear genome and play important roles in mitochondrial metabolism, showed decreasing expression during infection (Okano et al., 2001). These observations suggest that BmNPV infection does not affect mitochondria-specific transcription during BmNPV infection. So far, the effects of host gene expression and protein synthesis by NPV were examined in most cases using culture cells. It still remains unclear whether down-regulation of global protein gene expression and shutdown of global protein synthesis by NPV observed in culture cells (Mazzacano et al., 1999; Okano et al., 2001) also occur in vivo. Analyses of proteins other than antibacterial peptides in NPV-infected larvae are necessary to compare the results from NPV-infected cultured cells.

\section{ACKNOWLEDGEMENTS}

We thank Dr. Shogo Matsumoto at RIKEN (The Institute of Physical and Chemical Research) for providing a cysteine proteinase-deficient BmNPV mutant (orf 104). This work was supported in part by a Grant-in-Aid (Insect Technology Program) from the Ministry of Agriculture, Forestry and Fisheries of Japan.

\section{REFERENCES}

Chernysh, S., S. I. Kim, G. Bekker, V. A. Pleskach, N. A. Filatova, V. B. Anikin, V. G. Platino and P. Bullet (2002) Antiviral and antitumor peptides from insects. Proc. Natl. Acad. Sci. USA 99: 12628-12632.

Chowdhury, S., K. Taniai, S. Hara, K. Kadono-Okuda, Y. Kato, M. Yamamoto, J. Xu, S. K. Choi, N. C. Debnath, H. K. Choi, A. Miyanoshita, M. Sugiyama, A. Asaoka and M. Yamakawa (1995) cDNA cloning and gene expres- 
sion of lebocin, a novel member of antibacterial peptides from the silkworm, Bombyx mori. Biochem. Biophys. Res. Commun. 214: 271-278.

Clem, R. J., M. Fechheimer and L. K. Miller (1991) Prevention on apoptosis by a baculovirus gene during infection of insect cells. Science 254: 1388-1390.

De Gregorio, E., P. T. Spellman, P. Tzou, G. M. Rubin and B. Lemaitre (2002) The Toll and Imd pathways are the major regulators of the immune response in Drosophila. EMBO J. 21: 2568-2579.

Furukawa, S., H. Tanaka, H. Nakazawa, J. Ishibashi, T. Shono and M. Yamakawa (1999) Inducible gene expression of moricin, a unique antibacterial peptide from the silkworm (Bombyx mori). Biochem. J. 340: 265-271.

Furukawa, S., K. Taniai, J. Ishibashi, S. Hara, T. Shono and M. Yamakawa (1997) A novel member of lebocin gene family from the silkworm, Bombyx mori. Biochem. Biophys. Res. Commun. 238: 769-774.

Hirai, M., O. Terenius, W. Li and I. Faye (2004) Baculovirus and dsRNA induce Hemolin, but no antibacterial activity, in Antheraea pernyi. Insect Mol. Biol. 13: 399-405.

Hoffmann, J. A., F. C. Kafatos, C. A. Janeway and R. A. Ezekowitz (1999) Phylogenetic perspectives in innate immunity. Science 284: 1313-1318.

Hultmark, D., H. Steiner, T. Rasmuson and H. G. Boman (1980) Insect immunity. Purification and properties of three inducible bactericidal proteins from hemolymph of immunized pupae of Hyalophola cecropia. Eur. J. Biochem. 106: 7-16.

Kadono-Okuda, K., K. Taniai, Y. Kato, E. Kotani and M. Yamakawa (1995) Effects of synthetic Bombyx mori cecropin B on the growth of plant pathogenic bacteria. $J$. Invertebr. Pathol. 65: 309-310.

Kato, Y., K. Taniai, H. Hirochika and M. Yamakawa (1993) Expression and characterization of cDNAs for cecropin $\mathrm{B}$, an antibacterial protein of the silkworm, Bombyx mori. Insect Biochem. Molec. Biol. 23: 285-290.

Kimbrell, D. A. (1991) Insect antibacterial proteins: not just for insects and against bacteria. BioEssays 13: 657663.

Kobayashi, M., H. Mori and T. Yaginuma (1985) Stimulation of acid protease activity in the isolated pupal abdomens of the silkworm, Bombyx mori, infected with nuclear polyhedrosis virus. J. Invertebr. Pathol. 46: 202-204.

Mazzacano, C. A., X. Du and S. M. Thiem (1999) Global protein synthesis shutdown in Autographa californica nucleopolyhedrovirus-infected Ld652Y cells is rescued by tRNA from uninfected cells. Virology 260: 222-231.

Morimoto, M., H. Mori, T. Otake, N. Uebe, N. Kunita, M. Niwa, T. Murakami and S. Iwanaga (1991) Inhibitory effect of tachyplesin I on the proliferation of human immunodeficiency virus in vitro. Chemotherapy 37: 206211.

Murakami, T., M. Niwa, F. Tokunaga and T. Miyata (1991) Direct virus inactivation of tachyplesin I and its isopeptides from horseshoe crab hemocytes. Chemotherapy 37: 327-334.

Nakazawa, H., E. Tsuneishi, K. M. Ponnuvel, S. Furukawa, A. Asaoka, H. Tanaka, J. Ishibashi and M. Yamakawa (2004)
Antiviral activity of a serine protease from the digestive juice of Bombyx mori larvae against nucleopolyhedrovirus. Virology 321: 154-162.

Nakazawa, H., F. Yukuhiro, S. Furukawa, A. Sagisaka, H. Tanaka, J. Ishibashi and M. Yamakawa (2003) Spontaneous synthesis of an antibacterial peptide linked to ecdysis in lepidopteran insects. J. Insect Biotechnol. Sericol. 72: $133-137$.

Ohkawa, T., K. Majima and S. Maeda (1994) A cyteine proteinase encoded by the baculovirus Bombyx mori nuclear polyhedrosis virus. J. Virol. 68: 6619-6625.

Okano, K., T. Shimada, K. Mita and S. Maeda (2001) Comparative expressed-sequence-tag analysis of differential gene expression profiles in BmNPV-infected BmN cells. Virology 282: 348-356.

Ooi, B. G. and L. K. Miller (1988) Regulation of host RNA levels during baculovirus infection. Virology 166: 515523.

Ponnuvel, K. M., H. Nakazawa, S. Furukawa, A. Asaoka, J. Ishibashi, H. Tanaka and M. Yamakawa (2003) A lipase isolated from the silkworm Bombyx mori shows antiviral activity against nucleopolyhedrovirus. J. Virol. 77: 10725-10729.

Sambrook, J. and D. W. Russel (2001) Molecular Cloning: A Laboratory Manual. 3rd ed. Cold Spring Harbor Laboratory Press, Cold Spring Harbor, NY.

Slack, J. M., J. Kuzio and P. Faulkner (1995) Characterization of $v$-cath, a cathepsin L-like proteinase expressed by the baculovirus Autographa californica multiple nuclear polyhedrosis virus. J. Gen. Virol. 76: 1091-1098.

Stöven, S., I. Ando, L. Kadalayil, Y. Engström and D. Hultmark (2000) Activation of the Drosophila NF- $\kappa \mathrm{B}$ factor relish by rapid endoproteolytic cleavage. EMBO J. 1: $347-352$.

Sugiyama, M., H. Kuniyoshi, E. Kotani, K. Taniai, K. KadonoOkuda, Y. Kato, M. Yamamoto, M. Shimabukuro, S. Chowdhury, J. Xu, S. K. Choi, H. Kataoka, A. Suzuki and M. Yamakawa (1995) Characterization of a Bombyx mori cDNA encoding a novel member of the attacin family of insect antibacterial proteins. Insect Biochem. Molec. Biol. 25: 385-392.

Sun, S.-C. and I. Faye (1992) CIF, an insect immunoresponsive factor with DNA-binding properties similar to NFкB. Eur. J. Biochem. 204: 885-892.

Suzuki, T., T. Kanaya, H. Okazaki, K. Ogawa, A. Usami, H. Watanabe, K. Kadono-Okuda, M. Yamakawa, H. Sato, H. Mori, S. Takahashi and K. Oda (1997) Efficient protein production using a Bombyx mori nuclear polyhedrosis virus lacking the cysteine proteinase gene. J. Gen. Virol. 78: 3073-3080.

Taniai, K., S. Furukawa, T. Shono and M. Yamakawa (1996) Elicitors triggering the simultaneous gene expression of antibacterial proteins of the silkworm, Bombyx mori. Biochem. Biophys. Res. Commun. 226: 783-790.

Taniai, K., K. Kadono-Okuda, Y. Kato, M. Yamamoto, M. Shimabukuro, S. Chowdhury, J. Xu, E. Kotani, S. Tomino and M. Yamakawa (1995) Strucuture of two cecropin B-encoding genes and bacteria-inducible DNA-binding proteins which bind to the 5 '-upstream regulatory region 
in the silkworm, Bombyx mori. Gene 163: 215-219.

Wachinger, M., A. Kleinshmidt, D. Winder, N. von Pechmann, A. Ludvigsen, M. Neumann, R. Holle, B. Salmons, V. Erfle and R. Brack-Werner (1998) Antimicrobial peptides melitin and cecropin inhibit replication of human immunodeficiency virus I by suppressing viral gene expression. J. Gen. Virol. 79: 731-740.

Washburn, J. O., B. A. Kirkpatrick and L. E. Volkman (1996)
Insect protection against viruses. Nature 383: 767.

Yamakawa, M. and H. Tanaka (1999) Immune proteins and their gene expression in the silkworm, Bombyx mori. Dev. Comp. Immunol. 23: 281-289.

Zoog, S. J., J. J. Schiller, J. A. Wetter, N. Chejanovsky and P. D. Frieses (2002) Baculovirus apoptotic suppressor P49 is a substrate inhibitor of initiator caspases resistant to P35 in vivo. EMBO J. 21: 5130-5140. 